\title{
Organic-Inorganic Composites of Semiconductor Nanocrystals for Efficient Excitonics
}

\author{
Burak Guzelturk and Hilmi Volkan Demir* \\ Department of Electrical and Electronics Engineering, Department of Physics, UNAM - Institute of Materials Science and \\ Nanotechnology, Bilkent University, Ankara 06800, Turkey
}

LUMINOUS! Center of Excellence for Semiconductor Lighting and Displays, TPI - The Photonics Institute, School of Electrical and Electronic Engineering, School of Physical and Materials Sciences, Nanyang Technological University, Singapore 639798, Singapore

\begin{abstract}
Nanocomposites of colloidal semiconductor nanocrystals integrated into conjugated polymers are the key to soft-material hybrid optoelectronics, combining advantages of both plastics and particles. Synergic combination of the favorable properties in the hybrids of colloidal nanocrystals and conjugated polymers offers enhanced performance and new functionalities in light-generation and light-harvesting applications, where controlling and mastering the excitonic interactions at the nanoscale are essential. In this Perspective, we highlight and critically consider the excitonic interactions in the organic-inorganic nanocomposites to achieve highly efficient exciton transfer through rational design of the nanocomposites. The use of strong excitonic interactions in optoelectronic devices can trigger efficiency breakthroughs in hybrid optoelectronics.
\end{abstract}

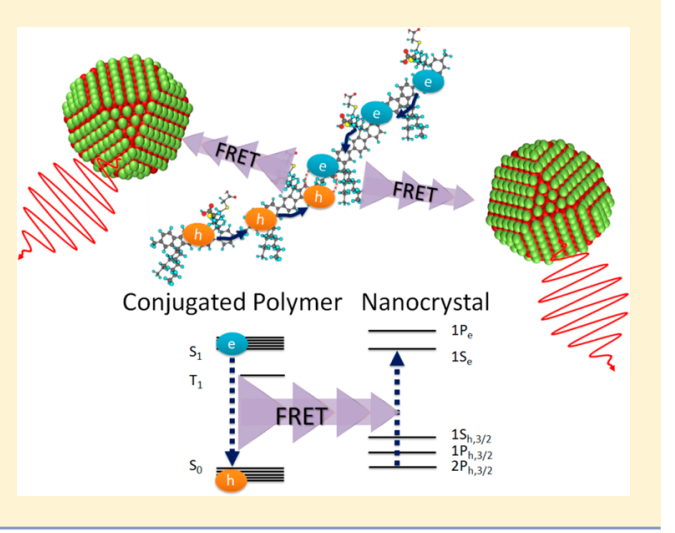

B oth colloidal semiconductor nanocrystals (NCs) and $\pi$ conjugated semiconductor polymers (CPs) are attractive materials for a broad range of applications including bioimaging, ${ }^{1,2}$ sensing, ${ }^{3,4}$ electronics, ${ }^{5,6}$ and photonics. ${ }^{7,8}$ Using these emerging materials for energy-efficient lighting, display and photovoltaic technologies have gained escalating interest in the past 2 decades. $^{9-12}$ To this end, combining favorable optical properties of the colloidal semiconductor NCs and versatile physical properties of the conjugated polymers in hybrid platforms offers enabling opportunities for highperformance devices along with new functionalities. For this purpose, in the organic-inorganic composite systems, controlling the photophysical properties becomes crucial. These properties principally comprise excitons and excitonic processes, which include exciton formation, diffusion, transfer, and dissociation as well as radiative and nonradiative recombination of the excitons. These excitonic processes are schematically depicted in Figure 1.

An exciton, which is a Coulombically bound electron-hole pair, is the primary form of the excited-state energy in the CPs and the NCs. An exciton can be created via either optical or electrical excitation (Figure 1a). Optical excitation occurs through absorption of a photon, while electrical excitation requires simultaneous injection of an electron and a hole. Within the lifetime of an exciton, it may radiatively or nonradiatively recombine (Figure $1 \mathrm{~b}$ ). Radiative recombination results in emission of a photon, whereas nonradiative recombination does not produce light but heat. Exciton diffusion (Figure 1c) is another process that is widely observed in the close-packed solid films of the CPs and the colloidal NCs. In the case of CPs, exciton diffusion/migration can happen either via exciton hopping within the delocalized excited-state landscape of $\mathrm{CP}^{13}$ or via Förster resonance energy transfer (FRET) between different chromophoric units of the CP. ${ }^{14,15}$ In the colloidal NCs, exciton diffusion dominantly takes place through long-range nonradiative energy transfer because excitons are confined to the NCs. ${ }^{16,17}$ The exciton diffusion length in the CPs and the NCs is typically on the order of $10 \mathrm{~nm} \cdot{ }^{10,17}$ For a solar cell, attaining a longer exciton diffusion length is desired for enhanced light-harvesting performance. ${ }^{18,19}$ In the case of a light-emitting diode (LED), exciton diffusion is undesired because it can cause trapping of the excitons at the trap/defect sites (Figure 1c), causing nonradiative recombination.

\section{Hybrids of conjugated polymers- colloidal nanocrystals are needed to boost the excitonic interac- tions without suffering from phase segregation.}

At the interfaces of materials having energetically staggered band alignment, excitons might dissociate through charge transfer. This process involves spatial overlap of the wave functions between different materials that are in close proximity $(<2 \mathrm{~nm})$ (Figure 1d). Thus, exciton dissociation leads to

Received: February 26, 2015

Accepted: May 28, 2015

Published: May 28, 2015 
(a) Exciton formation

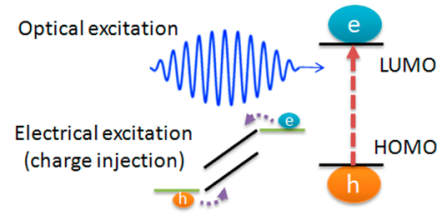

(d) Exciton dissocation

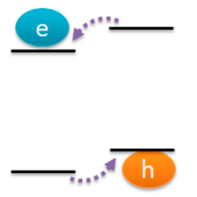

(b) Radiative/nonradiative recombination

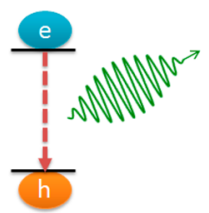

(c) Exciton diffusion
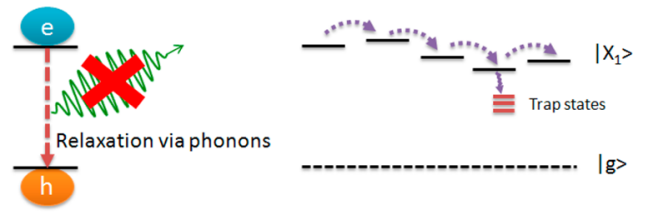

e) Excitation energy transfer (FRET and Dexter)
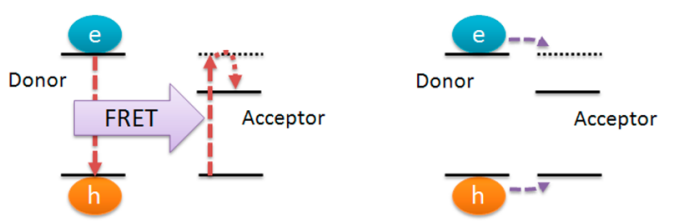

Figure 1. Excitonic processes are schematically summarized: (a) exciton formation, (b) radiative and nonradiative recombination, (c) exciton diffusion, (d) exciton dissociation, and (e) excitation energy transfer (FRET and Dexter energy-transfer mechanisms). (HOMO: highest occupied molecular orbital level; LUMO: lowest occupied molecular orbital level, $\left|\mathrm{X}_{1}\right\rangle$ represents the exciton state, $|\mathrm{g}\rangle$ is the ground state.)

reactive species through formation of free charge carriers, which finds use in photovoltaics for charge separation. On the other hand, in electroluminescent devices, it is essential to control the recombination zone of the excitons within which they radiatively recombine. The management of the exciton recombination zone can be achieved through excitation energy transfer (Figure 1e) including FRET, ${ }^{20}$ which is a near-field dipole-dipole coupling between the species that are in close proximity (typically $<10 \mathrm{~nm}$ ), and Dexter energy transfer, ${ }^{21}$ which is an electron exchange interaction between two species that are in intimate contact $(<2 \mathrm{~nm})$.

For the nonradiative energy-transfer mechanisms to occur, there must be a spectral overlap between the donor emission and the acceptor absorption to satisfy the resonance condition. ${ }^{22}$ Additionally, Dexter energy transfer requires the spatial overlap of the wave functions between the donoracceptor pair, whereas FRET does not require this because it is a long-range process. Both nonradiative energy-transfer mechanisms preserve the neutrality of the donor and the acceptor materials because excitons are being transferred instead of free carriers. The spatial extent of the colloidal $\mathrm{NCs}$ and the CPs, at least in one dimension, is less than $10 \mathrm{~nm}$, making FRET a versatile and efficient tool to control the excitons in the hybrid organic-inorganic composites. Yet, only singlet excitons can be transferred through FRET because the transition dipole in the donor must have a nonzero oscillator strength. Nevertheless, energy transfer between nonemissive triplet states can happen via Dexter energy transfer. ${ }^{23}$

Colloidal quantum dots (QDs), the NCs having threedimensional quantum confinement, have been shown to be highly attractive materials for light generation, owing to their spectrally narrow and efficient photoluminescence features. ${ }^{24,25}$ However, charge injection and transport has been severely hindered in the solid films of these QDs due to their bulky organic ligands and large-band-gap inorganic shells. ${ }^{26-28}$ Although it is possible to replace these bulky ligands with shorter ones ${ }^{29}$ or with inorganic halides, ${ }^{30,31}$ these processes mostly cause loss of photoluminescence due to poor surface passivation and introduced surface traps. Alternatively, CPs have been shown to exhibit electroluminescence since the late 1980 s, opening up the field of organic LEDs (OLEDs). ${ }^{32,33}$ The CPs exhibit better charge injection and transport properties as compared to the colloidal QDs. ${ }^{5,26}$ Yet, it is more difficult to tune the spectral emission in the CPs. ${ }^{34}$ Also, the CPs typically have much broader spectral emission widths, limiting their color purity in the OLEDs. ${ }^{35}$ These challenges have stimulated the use of organic-inorganic hybrid systems to synergistically integrate or combine CPs and QDs. The potential of the organic-inorganic hybrids has led the scientific community to delve deeper into their excitonic properties to tailor these materials for optimized light-generation and light-harvesting performance. $^{11,26,36,37}$

Exciton transfer from an organic semiconductor into colloidal QDs has been elucidated to be a favorable mechanism to excite the QDs in the LEDs. ${ }^{23,38-41}$ Such exciton transfer via nonradiative energy transfer has been investigated in several $\mathrm{CP}-\mathrm{QD}$ blends or hybrids by various reports ${ }^{40-51}$ and several reviews. $^{52-55}$ The QDs and CPs used in these reports are summarized in Table 1 . As one of the early reports, Anni et al.

Table 1. List of Colloidal NCs and Conjugated Polymers Used in Their Exciton-Transferring Blends and/or Hybrid Composites

\begin{tabular}{lll}
\multicolumn{1}{c}{ NC } & \multicolumn{1}{c}{ conjugated polymer } & \multicolumn{1}{c}{ ref } \\
$\begin{array}{l}\text { InAs/ZnSe (core/shell } \\
\text { QDs) }\end{array}$ & MEH-PPV and F6BT & 57 \\
PbS (core-only QDs) & MEH-PPV and/or CN-PPP & $41,43,58$ \\
CdSe (core-only QDs) & OPV and OPE & 45,59 \\
CdTe (core-only QDs) & PDFD (water-soluble, positively & $48-50$ \\
& charged) & 60 \\
CdSe/ZnS (core/shell & PFH derivatives (amine \\
QDs) & functionalized) & \\
& PDFC & 42 \\
& PF2/6 & 51,61 \\
& PSF & 47 \\
CdSe/CdZnSeS/ZnS & PTA- $b$-CAA and PTA- $b$-PFP & 62,63 \\
(core/alloyed-shell QDs) & (thiol functionalized) & 64 \\
& PF-CMSP (carboxyl & \\
& functionalized) & \\
\hline
\end{tabular}

have observed nonradiative energy transfer, which was suggested to be FRET, in the blended CP-QD films via time-resolved fluorescence spectroscopy, revealing the spectroscopic evidence of FRET from the CP into the QD. ${ }^{42}$ Previously, FRET from the organic dyes into the QDs has not been observed, which was attributed to the considerably faster photoluminescence lifetimes of the organic dyes. ${ }^{56}$ However, the CPs have been shown that they can be exciton 

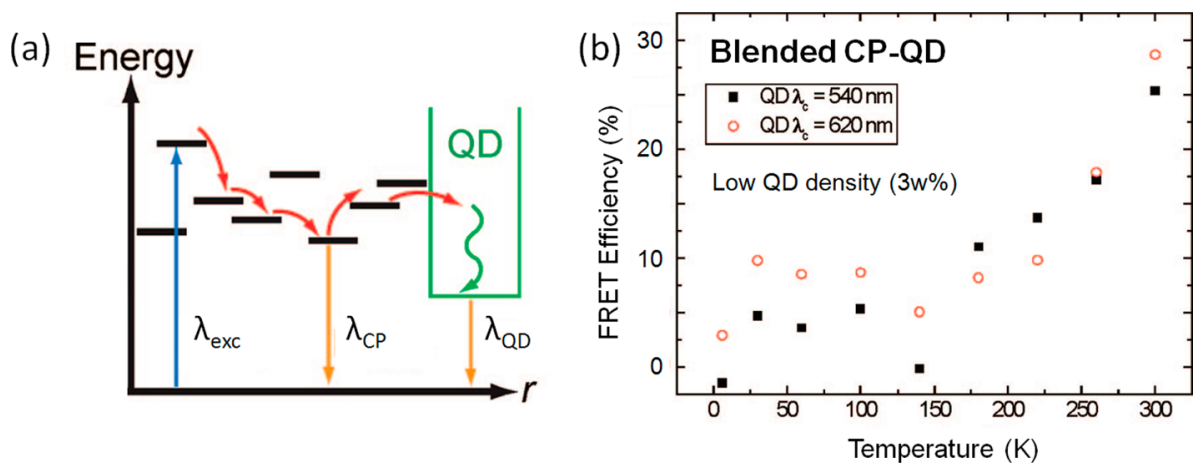

Figure 2. (a) Schematic demonstration of exciton-diffusion-assisted nonradiative energy transfer from a conjugated polymer into a colloidal QD. An exciton generated in the disordered density of states of a conjugated polymer can diffuse via hopping with the help of activation energy $(k T)$. Exciton diffusion assists nonradiative energy transfer to a QD from the conjugated polymer. (b) Temperature-dependent nonradiative energy-transfer efficiencies in the blended CP-QD films having a QD density of 3 wt \%. Adapted with permission from ref 51. Copyright 2009, American Chemical Society.
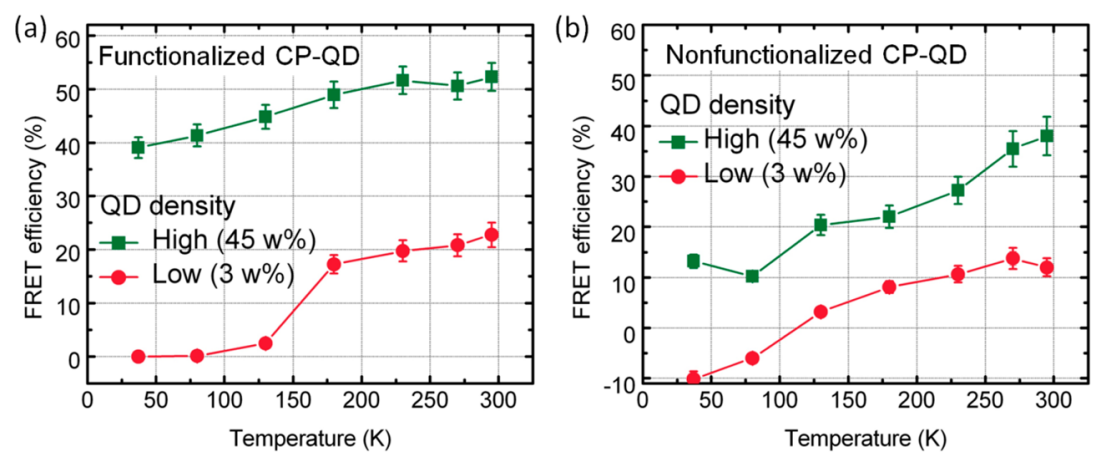

Figure 3. Nonradiative energy-transfer efficiencies as a function of temperature for (a) functionalized CP-QD hybrid and (b) nonfunctionalized $\mathrm{CP}-\mathrm{QD}$ blended films at low (3 wt \%) and high (45 wt \%) QD densities within in the polymer matrix. Reproduced from ref 40 with permission from The Royal Society of Chemistry.

donors for the QDs. This nonradiative energy transfer from the $\mathrm{CP}$ into the $\mathrm{QD}$ was also verified by photoluminescence excitation (PLE) spectroscopy. ${ }^{41}$ PLE of the QD emission was shown to exhibit strong enhancement in the spectral range, where the $\mathrm{CP}$ has strong absorption, owing to the exciton transfer from the CPs into the QDs. Other evidence of this type of exciton transfer was shown by the slower rise time of the fluorescence decay in the acceptor QDs due to exciton feeding from the CPs. ${ }^{46}$ Different from the early works, which generally focused on blended CP-QD films, Lutich et al. have investigated nonradiative energy transfer in an electrostatically integrated CP-QD hybrid. ${ }^{48}$ They have demonstrated that FRET is the dominant excitonic mechanism among others (Dexter energy transfer and charge transfer), with an efficiency as high as $80 \%$ despite a type-II band alignment between the $\mathrm{CP}$ and the QD. ${ }^{48}$

Later, Stöferle et al. put forward that exciton diffusion is crucially needed for nonradiative energy transfer to take place from the CPs into the QDs. ${ }^{51}$ With the help of exciton diffusion in the organic part, excitons would become effectively closer to the acceptor QDs (see Figure 2a) so that nonradiative energy transfer would be more efficient. ${ }^{65,66}$ In this aforementioned work, exciton-diffusion-assisted exciton transfer was demonstrated in blended CP-QD films by measuring the transfer efficiencies as a function of temperature. At low temperatures, exciton-transfer efficiency was found to be suppressed (see Figure $2 \mathrm{~b}$ ) due to temperature deactivation of the exciton diffusion in the CPs. Thus, at low temperatures, exciton diffusion could not assist exciton transfer. However, ref 51 considered only blended CP-QD films having QD density as low as $3 \mathrm{wt} \%$. This low density was intentionally set to prevent possible phase segregation, which is a common phenomenon in the blended CP-QD films. ${ }^{26,40,62,63}$

At increased QD densities in a polymer matrix, the QDs will form micron-scale aggregates in this polymer matrix. The phase segregation is detrimental against efficient nonradiative energy transfer because the donor CPs and the acceptor QDs are physically separated. Eventually, crucially exciton-diffusionassisted exciton transfer in the CP-QD composites was restricted with the observation only in blends incorporating low QD density, where exciton transfer is not strong. The interplay between exciton diffusion and exciton transfer had not been understood in the hybrid CP-QD nanocomposites. To this end, we have developed hybrid $\mathrm{CP}-\mathrm{QD}$ nanocomposites using functionalized CPs that have anchor groups facilitating strong attraction to the QD surfaces; thus, these hybrids substantially suppress phase segregation even for the QD densities up to 80 wt $\%{ }^{40}$

Previously, different hybridization methods have been used to integrate the QDs and the CPs together, including ligand exchange of QDs with CPs or synthesizing QDs in a CP-based ligand medium. ${ }^{55,67,68}$ These methods generally do not preserve high-quality optical properties of the QDs and may cause exciton dissociation and charge transfer due to intimate contact between the organic and inorganic species. ${ }^{45}$ Therefore, using core/shell QDs together with functionalized CPs having anchor 

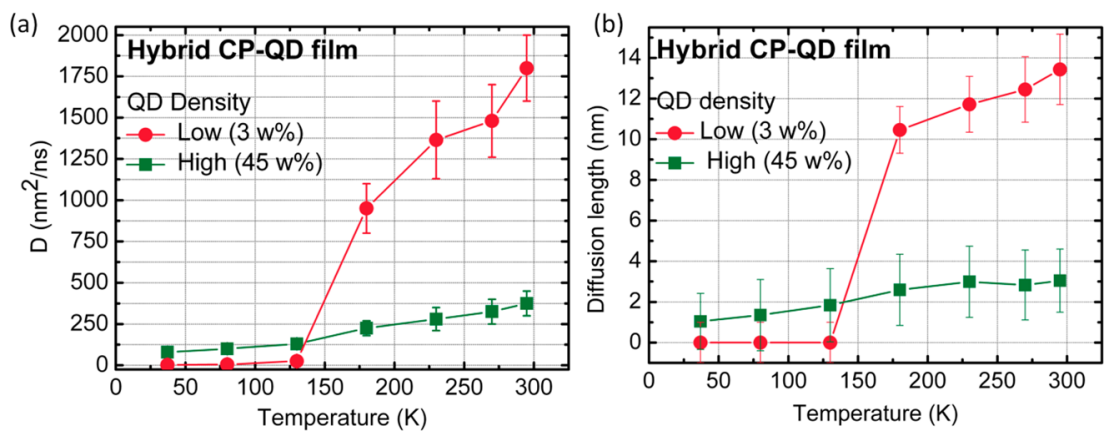

Figure 4. Temperature-dependent (a) exciton diffusion coefficient and (b) exciton diffusion length in the functionalized CP when the polymer matrix incorporates low (3 wt \%) and high (45 wt \%) density QDs. Reproduced from ref 40 with permission from The Royal Society of Chemistry.

groups stands out as an attractive route to boost exciton transfer in the hybrids while preserving the favorable optical properties of the QDs. To date, generally amine, ${ }^{60}$ carboxyl, ${ }^{40}$ and thiol ${ }^{62,63}$ groups (see Table 1) have been used in the functionalized CPs as the anchor groups. This type of hybridization will be promising for future hybrid organicinorganic optoelectronic systems.

In our previous work employing hybrids of the functionalized CPs and the QDs, we achieved phase-segregation free hybrids having QD densities up to 80 wt $\%{ }^{40}$ By this means, nonradiative energy transfer from the CPs into the QDs was shown to reach efficiencies as high as $80 \%$, which is a record high in the hybrid CP-QD films. Furthermore, we have studied nonradiative energy transfer in these hybrid $\mathrm{CP}-\mathrm{QD}$ nanocomposites as a function of temperature. For this, in the hybrids, we investigated two different QD densities, low (3 wt $\%)$ and high (45 wt \%). Also, as a negative control group, we used another $\mathrm{CP}$, which shares a similar polymer backbone but does not have any functional anchor group. These composites were dubbed as blended $\mathrm{CP}-\mathrm{QD}$ films, in which phase segregation would be unpreventable. Using time-resolved fluorescence spectroscopy, we calculated nonradiative energytransfer efficiencies in the hybrid and blended films, as shown in Figure $3 a$ and $b$, respectively. In the case of low QD density in the hybrid and the blend, exciton-transfer efficiencies were observed to considerably decrease as the temperature was decreased, as was previously observed by Stöferle et al. ${ }^{51}$ This suggests that exciton diffusion assistance is crucial for the low $\mathrm{QD}$ densities in the CP-QD composites, whether it is hybrid or a blend.

In the case of high QD density, however, we observe a sharp difference in temperature-dependent exciton-transfer efficiencies between the hybrid and the blend. For the hybrid film, the temperature-dependent exciton transfer is significantly reduced with efficiencies in the range of $40-50 \%$ as a function of temperature (see Figure 3a). On the other, for the blended film, energy-transfer efficiencies were found to change noticeably (from 40 to $10 \%$ ) in the same temperature range. These contrasting observations suggest that nonradiative energy transfer in the hybrid films incorporating high QD density do not crucially require exciton diffusion assistance. Most of the excitons are transferred directly into the QDs without the assistance of exciton diffusion. However, in the blended films having high QD density, exciton diffusion assistance is still needed due to phase segregation between the CP and the QDs, creating a large effective donor-acceptor distance.

To further elucidate the interplay between exciton diffusion and exciton transfer in the hybrid CP-QD nanocomposites, we have employed a model that takes into account exciton diffusion assistance into the donor fluorescence decay kinetics as suggested by Gösele et al., ${ }^{69}$ similar to the Yokota-Tanimoto model. ${ }^{51,66}$ To match the experimental exciton-transfer rates in the hybrid films, exciton diffusion coefficients were predicted by the model, as shown in Figure 4a. In the case of low QD density in the hybrid, the exciton diffusion coefficient was found to be $\sim 1750 \mathrm{~nm}^{2} / \mathrm{ns}$ at room temperature, which is in good agreement with the previously reported diffusion coefficient $\left(1440 \mathrm{~nm}^{2} / \mathrm{ns}\right)$ for a similar conjugated polymer. ${ }^{70}$ As the temperature is decreased, the diffusion coefficient is observed to decrease and is severely suppressed below $150 \mathrm{~K}$. There might be a phase change in the CP below this temperature, restricting the exciton diffusion, as observed before for a similar type of conjugated polymers. ${ }^{51,66}$

In the case of high QD density incorporating hybrid, the exciton diffusion coefficient was found to be $\sim 375 \mathrm{~nm}^{2} / \mathrm{ns}$ at room temperature, which is almost an order of magnitude lower as compared to that in the hybrid having low QD density. Therefore, exciton diffusion is significantly slowed down in the hybrids of high QD density. We attribute this impeded exciton diffusion to the fact that two different exciton diffusion mechanisms exist in the conjugated polymers, fast interchain and slow intrachain exciton diffusion, as was previously revealed. ${ }^{14,71}$ By introducing a large number of QDs into the polymer, one would expect to suppress fast interchain exciton diffusion due to the increased chain-to-chain distance in the presence of the incorporated QDs. Previously, intrachain exciton diffusion was also found to be an order of magnitude slower as compared to the interchain exciton diffusion, which is also in good quantitative agreement with the observation of slowing exciton diffusion. ${ }^{71}$

Furthermore, we calculated exciton diffusion lengths in the CP-QD hybrids. The hybrid having low QD density exhibited an exciton diffusion length of $\sim 15 \mathrm{~nm}$ at room temperature, which represents a good match with the previous literature. ${ }^{72}$ As temperature is decreased, the exciton diffusion length decreases and becomes very short below $150 \mathrm{~K}$ due to temperature deactivation of the exciton diffusion and possible phase change in the polymer. ${ }^{51,66}$ On the other hand, the hybrid having high QD density exhibited much a smaller exciton diffusion length of $\sim 2.5 \mathrm{~nm}$ at room temperature, which is 6-fold smaller than that observed in the hybrids having low QD density. Thus, the exciton diffusion length is decreased with increasing QD density in the hybrids. The observed exciton diffusion length of $2.5 \mathrm{~nm}$ in the high QD density hybrid agrees well with the fast downhill exciton diffusion in the conjugated polymers. ${ }^{73}$ These observations support that 
exciton diffusion assistance to nonradiative energy transfer in the $\mathrm{CP}-\mathrm{QD}$ hybrids is not crucial anymore if high $\mathrm{QD}$ density is used in the hybrid. This shows a major characteristic difference as compared to the blended CP-QD films, where exciton diffusion assistance is always crucial. Thus, a direct exciton-transfer channel, which would not need exciton diffusion assistance, prevails in the hybrid $\mathrm{CP}-\mathrm{QD}$ nanocomposites, as observed by temperature-insensitive exciton transfer and suppressed exciton diffusion in the hybrid CP-QD nanocomposites.

\section{Exciton diffusion is not crucially needed in high QD density hybrids, in contrast to blends and low QD density hybrids. A direct exciton-transfer channel can pre- vail in the $C P-Q D$ hybrids.}

An independent support to this view comes from another class of hybrid $\mathrm{CP}-\mathrm{QD}$ nanocomposites that have been constructed via self-assembling monolayers of positively charged semiconducting CP and negatively charged QDs in an alternating order (using layer-by-layer assembly). ${ }^{49}$ The electrostatically integrated organic-inorganic hybrids (for which the QD density was estimated to be 18 wt \%) also exhibited FRET efficiencies that are insensitive to temperature. Again, suppression of the exciton diffusion in the CP was observed indirectly through suppressed defect emission in the $\mathrm{CPs}^{49}$ Figure 5 schematically illustrates the fundamental interplay between the exciton diffusion and the exciton transfer in the blended and the hybrid composites.

Additionally, the ligands of the QDs play an important role for FRET efficiencies in the organic-inorganic nanocomposites. Previously, it has been shown that PbS QDs with shorter ligands could enhance FRET efficiencies up to 3-folds in their blended composites with MEH-PPV. ${ }^{41}$ Therefore, shorter ligands can be employed to facilitate faster and more efficient nonradiative energy transfer because the donor-acceptor separation can be effectively made shorter. However, recent studies have shown that the morphology of the ligands on the QD surfaces can also be critical for the excitonic interactions. The nanoscale morphology of the ligands (conformation, bundling, interpenetration, etc.) on the surface of the CdSe QDs has been elucidated to strongly suppress the charge transfer in the $\mathrm{CP}-\mathrm{QD}$ composites. ${ }^{74}$

LEDs that combine the conjugated polymers and the colloidal QDs together as the emissive layers have been under research for the past 2 decades. ${ }^{11,40,47,57,58,63,75,76}$ Colvin et al. first demonstrated that it is possible to achieve electroluminescence from the QDs using the composites of the QDs incorporated into the conjugated polymer. ${ }^{11}$ Such LEDs having blended CP-QD films have been shown to be promising for near-infrared (NIR) and infrared (IR) emission. ${ }^{57,75}$ In these blended LEDs, excitation of the QDs via nonradiative energy transfer from the $C P$ has been suggested to be dominant pumping mechanism as compared to charge injection pumping. However, these LEDs based on blended polymer-QD films always exhibit mixed emission from both the $\mathrm{CP}$ and the $\mathrm{QD}$ in their electroluminescence spectra due to incomplete exciton transfer from the CPs. ${ }^{11,47,57,76}$ The mixed emission has been favorably utilized for white light generation in the blended CP-QD films. ${ }^{77,78}$ Yet, complete exciton transfer is highly desired to achieve spectrally pure LEDs (i.e., with color purity), which can be realized only using hybrid $\mathrm{CP}-\mathrm{QD}$ films having high $\mathrm{QD}$ density. ${ }^{40}$ Previously, ref 62 also demonstrated CP-QD hybrids exhibiting dominant QD emission (see Figure 6c for hybrid LEDs having increasing QD loading in the hybrids from 30 wt $\%$ for $\mathrm{H} 1$ up to 70 wt $\%$ for $\mathrm{H} 5)$ in the electroluminescence spectrum of the hybrid LEDs owing to the suppressed phase segregation through anchor groups in the polymer (see Figure 6a and b).

In our previous work, we have demonstrated excitonically driven hybrid LEDs, dubbed as XLEDs, ${ }^{40}$ based on the functionalized CP-QD hybrids employing QD densities larger than $70 \mathrm{wt} \%$. This ensures highly efficient direct transfer of the excitons in the hybrid due to absence of phase segregation. Figure $6 \mathrm{~d}$ shows the electroluminescence spectra of the XLEDs having different color-emitting QDs. Also, XLEDs offer high color purity with dominant $\mathrm{QD}$ emission at increased QD density in the hybrid owing to the boosted exciton transfer (Figure 6e). Compared to their control group LEDs using only the QDs in the emissive layer, XLEDs exhibit a significant enhancement in external quantum efficiency (an order of magnitude improvement in this implementation here). ${ }^{40}$ These reports suggest that it is possible to combine the efficient

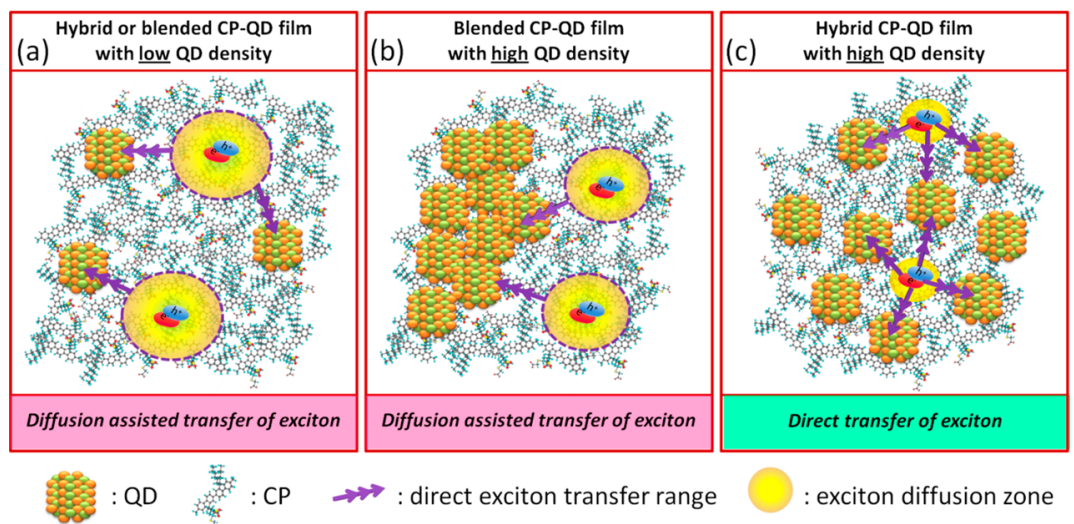

Figure 5. Exciton-diffusion-assisted exciton-transfer mechanism in (a) a low density QD incorporating hybrid or blend film and (b) a high density QD incorporating blended film. Direct exciton-transfer mechanism in (c) a high density QD incorporating hybrid. Reproduced from ref 40 with permission from The Royal Society of Chemistry. 
(a)
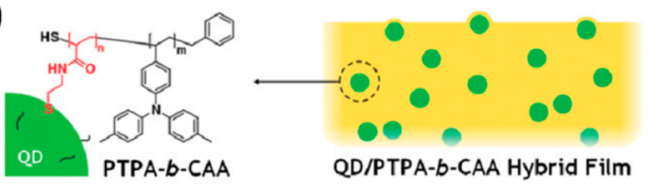

(b)

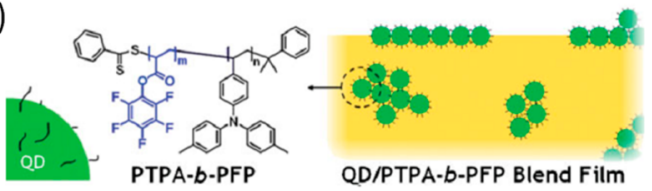

(d)

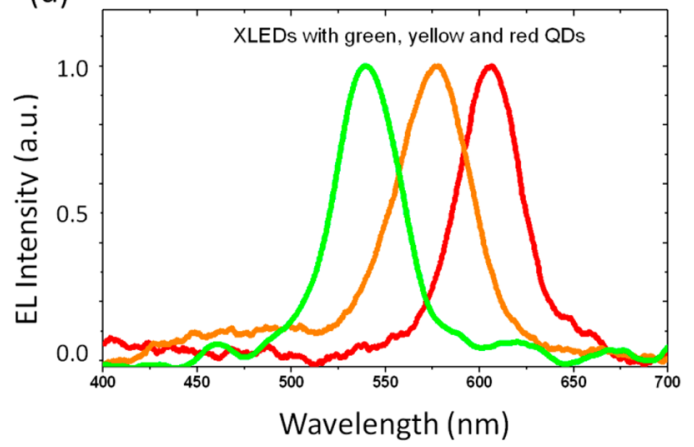

(c)

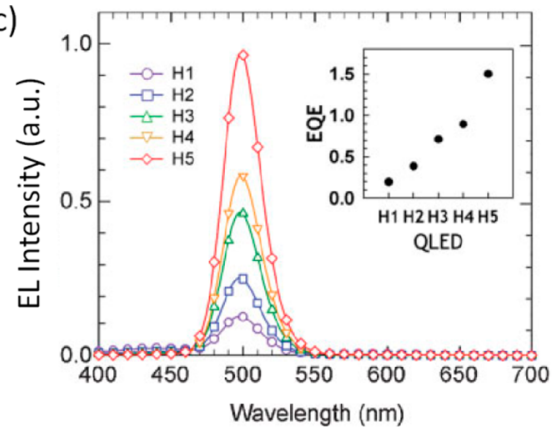

(e)

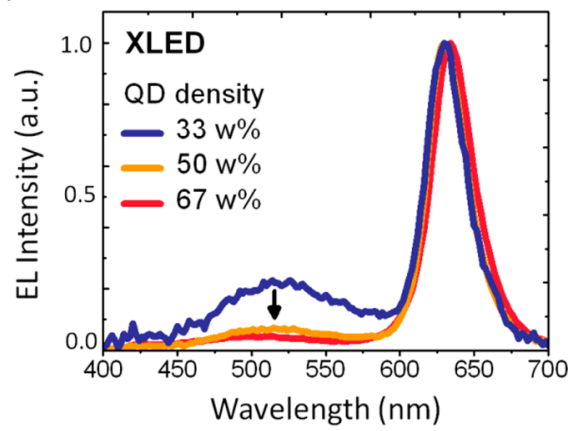

Figure 6. (a) Hybrid CP-QD and (b) blended CP-QD films. (c) Electroluminescence spectra of the hybrid LEDs (H1-H5) demonstrating only green $\mathrm{QD}$ emission (the QD loading concentration increases from 30 to $70 \mathrm{wt} \%$ from H1 to H5). (d) Excitonically driven LEDs (XLEDs) using hybrid CP-QD films incorporating green-, yellow-, and red-emitting QDs. (e) XLEDs with increasing QD density in the hybrid CP-QD film showing almost complete QD emission in the electroluminescence. $(\mathrm{a}-\mathrm{c}$ ) Adapted with permission from ref 62, Copyright 2009, John Wiley \& Sons, Inc.

charge injection through the CPs and almost complete exciton transfer into the QDs using tailored hybrid organic-inorganic excitonic nanocomposites.

Here, it is also worth noting that, although excitonic pumping of the QDs is very useful toward enhanced hybrid LEDs, there is a fundamental limitation arising due to nonemissive triplet states in the fluorescent CPs. According to spin statistics, $3 / 4$ of the excitons formed in a fluorescent semiconductor organic material through electrical excitation will be triplet-state excitons, which are spin-forbidden nonemissive states. ${ }^{79}$ This phenomenon restricts the performance of the LEDs employing fluorescent organic materials. ${ }^{80}$ It is not possible to harvest triplet-state excitons by transferring them to the QDs via FRET because triplet-state excitons have zero oscillator strength. An alternative method is using phosphorescent organic materials, where triplet states become emissive due to strong spin-orbit coupling. ${ }^{79}$ In such phosphorescent organic media, nonradiative energy transfer becomes viable too. 81,82

In our recent work, we have shown that exciton transfer from phosphorescent small organic molecules into core/shell QDs can enhance the fluorescence of the QDs by 3-fold, as revealed by time-resolved fluorescence and PLE spectroscopy. ${ }^{82} \mathrm{We}$ have also employed a similar hybrid phosphorescent organicinorganic composite to harvest the triplet-state excitons in the hybrid LEDs. ${ }^{83}$ This was enabled by a smart design of the QD and phosphorescent organic-molecule-doped electron-transport layers that led us to achieve highly color pure LEDs with external quantum efficiencies as high as $2.1 \%$, resulting in an enhancement factor over 4-fold as compared to the LEDs that do not harvest the triplet excitons. ${ }^{83}$ This type of phosphorescent organic-inorganic hybrids exhibiting strong exciton transfer will be the key to energy-efficient hybrid light generation through rational design and optimization of the hybrid composites.

Another means to harness triplet-state excitons has been recently shown using Dexter energy transfer. Organic molecules of tetracene and pentacene were shown to transfer their nonemissive triplet-state excitons into NIR-emitting $\mathrm{PbS}$ or PbSe QDs with an efficiency as high as $90 \% .{ }^{84,85}$ Furthermore, this energy transfer was shown to be boosted via singlet exciton fission, which results in formation of two triplet excitons from a singlet exciton. This new route of harnessing the triplet-state excitons might advance both light-generation and lightharvesting performance in the hybrid organic-inorganic systems to address the efficiency challenge arising due to nonemissive triplet states in the organic semiconductors.

Organic-inorganic CP-QD composites are also attractive for light-harvesting applications. The observation of efficient exciton dissociation and charge separation at the CP-QD interfaces ${ }^{26}$ had strongly suggested that these hybrid material systems would be promising for photovoltaics. The demonstration of the first hybrid CP-QD solar cells came after following the early studies on the charge separation in the $\mathrm{CP}-$ QD hybrids. ${ }^{37}$ Since then, in the past decade, there have been various efforts to improve the performance of the hybrid solar cells, which are summarized in recent specialized reviews. ${ }^{86,87}$ In this field, the current research generally aims at achieving a fine control over the nanoscale morphology and nanoparticle surface chemistry to boost the charge generation and transport for a maximized solar cell performance. To this end, hybrids of CdS QDs and PH3T nanowires have been introduced, which advantageously benefit from the elongated morphology of the nanowires for enhanced photocurrent densities. ${ }^{88}$ A similar approach has been also pursued by using the nanorods and the nanowires of CdSe and CdS NCs. ${ }^{89}$ The underpinning goal in 
such morphologically controlled hybrids is to realize domain sizes that are comparable to the exciton diffusion lengths $(\sim 10$ $\mathrm{nm}$ ) in order to maximize the charge-generation efficiency. Recently, Z. Liu et al. have shown that vertical phase segregation can be also favorably used in the organic-inorganic composites of PbSeS QDs and PDTPBT hole-accepting CPs. ${ }^{90}$ In contrast to the excitonically driven hybrid LEDs, where phase segregation was shown to be highly detrimental for the color purity of the electroluminescence, ${ }^{40}$ the hybrids of $\mathrm{PbSeS}$ QDs-PDTPBT CPs can simultaneously combine the ultrafast charge separation and efficient charge transport through vertical phase segregation. $^{90}$ The device architecture together with the proposed vertical-phase-separated morphology is shown in Figure 7. These vertically phase-separated solar cells have

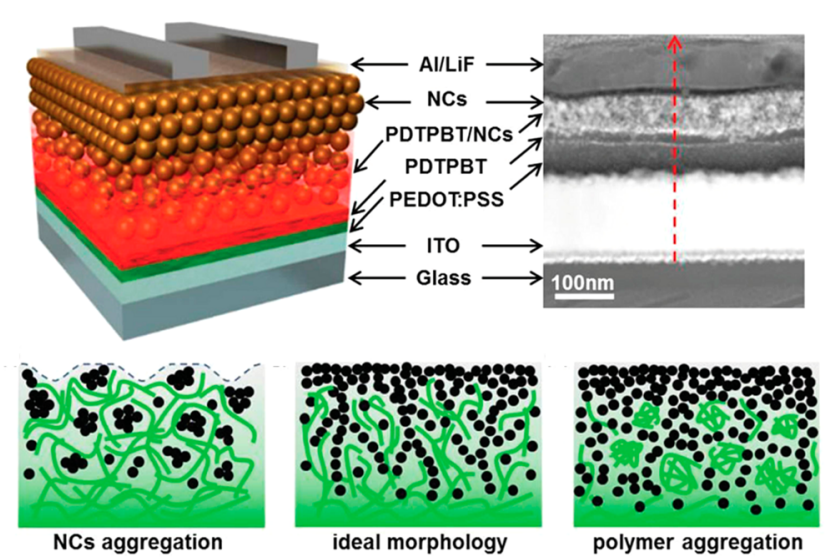

Figure 7. Vertical phase segregation ("ideal morphology") in the $\mathrm{PbSeS} \mathrm{QD}-\mathrm{PDTPBT} \mathrm{CP}$ hybrids has been shown to be very effective for achieving ultrafast charge separation and efficient charge transport to boost the performance of the hybrid solar cells. The optimization of the morphology control required one to avoid "NCs aggregation" and "polymer aggregation" via optimization of the concentrations of the QDs and the CPs. Adapted with permission from ref 90, Copyright 2013, John Wiley \& Sons, Inc.

achieved record-high power conversion efficiencies (as high as $5.5 \%) .{ }^{90}$ In addition to the controlled morphology in the hybrid nanocomposites, control of the surface chemistry of the NCs stands out as an effective means for controlling the exciton dissociation and charge transfer. To date, bundling of the ligands on the QD surfaces has been shown to effectively tailor the electron transfer from the QDs into the CPs. ${ }^{74}$ Similarly, the shell thickness of the core/shell QDs has been shown to be essential for the optimization of the charge-transfer processes in the composites of $\mathrm{CdSe} / \mathrm{ZnS}$ QDs-FHQ $\mathrm{CPs}^{91}$ and $\mathrm{PbS}$ QDs-PCPDTBT CPs. ${ }^{92}$

Alternative to the CPs, conjugated polymer nanoparticles (CPNs) have emerged in the past decade as attractive organic semiconductors for various applications owing to their large absorption cross section and high photoluminescence efficiency. ${ }^{93}$ The CPNs are also promising candidates for the hybrid organic-inorganic composites for enhanced lightgeneration and -harvesting purposes. Recent research efforts indicate that the hybrids of the CPNs incorporated with the CdTe QDs through electrostatic interactions can realize FRET efficiencies as high as $73 \% .{ }^{94}$ Exciton diffusion in the CPNs has been a subject of various recent studies. It has been shown that exciton diffusion in the CPNs is comparable to that of their $\mathrm{CP}$ counterparts with diffusion lengths of $\sim 10-12 \mathrm{~nm} .^{95,96}$
However, the temperature dependence of the exciton diffusion in the CPNs still has to be understood. In the near-future, $\mathrm{CPN}-$ based hybrids may become more interesting for highly excitonic hybrid nanocomposites. ${ }^{97}$

\section{To harness the triplet-state ex- citons for enhanced light gener- ation, either FRET in phosphor- escent organic-material-based hybrids or Dexter energy transfer in fluorescent organic-material- based hybrids can be used.}

In conclusion, controlling excitonic processes is essential in the organic-inorganic nanocomposites to use their full potential in light-generation and light-harvesting applications. To this end, we have presented the fundamental excitonic processes in the composite organic-inorganic systems of colloidal semiconductor NCs. We have critically highlighted the recent contributions on the understanding of the interplay between the exciton diffusion and exciton transfer in the hybrid nanocomposites. This comprehension will be important for designing excitonically driven hybrid LEDs for ultraefficient performance. Furthermore, we have indentified the challenge of the limited triplet-state harvesting in the organic-inorganic hybrids and highlighted the ongoing research with a future direction.

\section{AUTHOR INFORMATION}

\section{Corresponding Author}

*E-mail: volkan@bilkent.edu.tr or hvdemir@ntu.edu.sg.

\section{Notes}

The authors declare no competing financial interest.

\section{Biographies}

Burak Guzelturk is a Ph.D. candidate in Electrical and Electronics Engineering at Bilkent University, where he also earned his B.S. and M.S. degrees in the same department in 2009 and 2011, respectively. He has worked under the supervision of Prof. Demir in the Devices and Sensors Group at the National Nanotechnology Research Center (UNAM) at Bilkent University. His research is focused on the development of excitonically tailored colloidal semiconductor nanocrystals and their hybrids for high-efficiency optoelectronic technologies including light-emitting diodes and lasers.

Prof. Hilmi Volkan Demir obtained his M.S. and Ph.D. degrees in electrical engineering at Stanford University in 2000 and 2004, respectively. $\mathrm{He}$ is an NRF Fellow of Singapore and Nanyang Associate Professor at NTU Singapore and serves as the Director of the Luminous! Center of Excellence for Semiconductor Lighting and Displays. Concurrently, he is the EURYI Associate Professor at Bilkent University Turkey. His research work includes the science and technology of excitonics and plasmonics for light generation and harvesting and nanocrystal optoelectronics for semiconductor lighting. http://www.devicesandsensors.bilkent.edu.tr/

\section{ACKNOWLEDGMENTS}

The authors are grateful for the financial support from EU-FP7 Nanophotonics4Energy NoE and TUBITAK EEEAG 109E002, 109E004, 110E010, 110E217, 112E183, and 114E410 and also that from NRF-RF-2009-09, NRF-CRP-6-2010-02, and 
A*STAR of Singapore. H.V.D. acknowledges support from ESF-EURYI and TUBA-GEBIP.

\section{REFERENCES}

(1) Bruchez, M., Jr. Semiconductor Nanocrystals as Fluorescent Biological Labels. Science 1998, 281, 2013-2016.

(2) Gaylord, B. S.; Heeger, A. J.; Bazan, G. C. DNA Hybridization Detection with Water-Soluble Conjugated Polymers and Chromophore-Labeled Single-Stranded DNA. J. Am. Chem. Soc. 2003, 125, 896-900.

(3) Medintz, I. L.; Uyeda, H. T.; Goldman, E. R.; Mattoussi, H.; McQuade, D. T.; Pullen, A. E.; Swager, T. M. Quantum Dot Bioconjugates for Imaging, Labelling and Sensing. Nat. Mater. 2005, 4, 435-446.

(4) McQuade, D. T.; Pullen, A. E.; Swager, T. M. Conjugated Polymer-Based Chemical Sensors. Chem. Rev. 2000, 100, 2537-2574.

(5) Sirringhaus, H.; Brown, P. J.; Friend, R. H.; Nielsen, M. M.; Bechgaard, K.; Langeveld-Voss, B. M. W.; Spiering, A. J. H.; Janssen, R. A. J.; Meijer, E. W.; Herwig, P.; et al. Two-Dimensional Charge Transport in Self-Organized, High-Mobility Conjugated Polymers. Nature 1999, 401, 685-688.

(6) Talapin, D. V.; Murray, C. B. PbSe Nanocrystal Solids for n- and p-Channel Thin Film Field-Effect Transistors. Science 2005, 310, 8689.

(7) Talapin, D. V.; Lee, J.-S.; Kovalenko, M. V.; Shevchenko, E. V. Prospects of Colloidal Nanocrystals for Electronic and Optoelectronic Applications. Chem. Rev. 2010, 110, 389-458.

(8) Sirringhaus, H. Integrated Optoelectronic Devices Based on Conjugated Polymers. Science 1998, 280, 1741-1744.

(9) Friend, R. H.; Gymer, R. W.; Holmes, A. B.; Burroughes, J. H.; Marks, R. N.; Taliani, C.; Bradley, D. D. C.; Santos, D. A.; Dos Bredas, J. L.; Logdlund, M.; et al. Electroluminescence in Conjugated Polymers. Nature 1999, 397, 121-128.

(10) Günes, S.; Neugebauer, H.; Sariciftci, N. S. Conjugated Polymer-Based Organic Solar Cells. Chem. Rev. 2007, 107, 13241338.

(11) Colvin, V. L.; Schlamp, M. C.; Alivisatos, A. P. Light-Emitting Diodes Made from Cadmium Selenide Nanocrystals and a Semiconducting Polymer. Nature 1994, 370, 354-357.

(12) Gur, I.; Fromer, N. A.; Geier, M. L.; Alivisatos, A. P. Air-Stable All-Inorganic Nanocrystal Solar Cells Processed from Solution. Science 2005, 310, 462-465.

(13) Collini, E.; Scholes, G. D. Coherent Intrachain Energy Migration in a Conjugated Polymer at Room Temperature. Science 2009, 323, 369-373.

(14) Beljonne, D.; Pourtois, G.; Silva, C.; Hennebicq, E.; Herz, L. M.; Friend, R. H.; Scholes, G. D.; Setayesh, S.; Mullen, K.; Bredas, J. L. Interchain vs. Intrachain Energy Transfer in Acceptor-Capped Conjugated Polymers. Proc. Natl. Acad. Sci. U.S.A. 2002, 99, 1098210987.

(15) Brédas, J.-L.; Beljonne, D.; Coropceanu, V.; Cornil, J. ChargeTransfer and Energy-Transfer Processes in $\pi$-Conjugated Oligomers and Polymers: A Molecular Picture. Chem. Rev. 2004, 104, 49715004.

(16) Kagan, C.; Murray, C.; Nirmal, M.; Bawendi, M. Electronic Energy Transfer in CdSe Quantum Dot Solids. Phys. Rev. Lett. 1996, $76,1517-1520$.

(17) Crooker, S.; Hollingsworth, J.; Tretiak, S.; Klimov, V. Spectrally Resolved Dynamics of Energy Transfer in Quantum-Dot Assemblies: Towards Engineered Energy Flows in Artificial Materials. Phys. Rev. Lett. 2002, 89, 186802.

(18) Xing, G.; Mathews, N.; Sun, S.; Lim, S. S.; Lam, Y. M.; Grätzel, M.; Mhaisalkar, S.; Sum, T. C. Long-Range Balanced Electron- and Hole-Transport Lengths in Organic-Inorganic $\mathrm{CH}_{3} \mathrm{NH}_{3} \mathrm{PbI}_{3}$. Science 2013, 342, 344-347.

(19) Guzelturk, B.; Erdem, O.; Olutas, M.; Kelestemur, Y.; Demir, H. V. Stacking in Colloidal Nanoplatelets: Tuning Excitonic Properties. ACS Nano 2014, 8, 12524-12533.
(20) Förster, T. Zwischenmolekulare Energiewanderung und Fluoreszenz. Ann. Phys. 1948, 437, 55-75.

(21) Dexter, D. L. A Theory of Sensitized Luminescence in Solids. J. Chem. Phys. 1953, 21, 836.

(22) Lakowicz, J. R. In Principles of Fluorescence Spectroscopy; Lakowicz, J. R., Ed.; Springer US: Boston, MA, 2006; Vol. 13.

(23) Agranovich, V. M.; Gartstein, Y. N.; Litinskaya, M. Hybrid Resonant Organic-Inorganic Nanostructures for Optoelectronic Applications. Chem. Rev. 2011, 111, 5179-5214.

(24) Murray, C. B.; Norris, D. J.; Bawendi, M. G. Synthesis and Characterization of Nearly Monodisperse CdE (E = Sulfur, Selenium, Tellurium) Semiconductor Nanocrystallites. J. Am. Chem. Soc. 1993, $115,8706-8715$.

(25) Alivisatos, A. P. Perspectives on the Physical Chemistry of Semiconductor Nanocrystals. J. Phys. Chem. 1996, 100, 13226-13239.

(26) Greenham, N.; Peng, X.; Alivisatos, A. Charge Separation and Transport in Conjugated-Polymer/Semiconductor-Nanocrystal Composites Studied by Photoluminescence Quenching and Photoconductivity. Phys. Rev. B 1996, 54, 17628-17637.

(27) Leatherdale, C.; Kagan, C.; Morgan, N.; Empedocles, S.; Kastner, M.; Bawendi, M. Photoconductivity in CdSe Quantum Dot Solids. Phys. Rev. B 2000, 62, 2669-2680.

(28) Morgan, N.; Leatherdale, C.; Drndić, M.; Jarosz, M.; Kastner, M.; Bawendi, M. Electronic Transport in Films of Colloidal CdSe Nanocrystals. Phys. Rev. B 2002, 66, 075339.

(29) Luther, J. M.; Law, M.; Song, Q.; Perkins, C. L.; Beard, M. C.; Nozik, A. J. Structural, Optical, and Electrical Properties of SelfAssembled Films of PbSe Nanocrystals Treated with 1,2-Ethanedithiol. ACS Nano 2008, 2, 271-280.

(30) Tang, J.; Kemp, K. W.; Hoogland, S.; Jeong, K. S.; Liu, H.; Levina, L.; Furukawa, M.; Wang, X.; Debnath, R.; Cha, D.; et al. Colloidal-Quantum-Dot Photovoltaics Using Atomic-Ligand Passivation. Nat. Mater. 2011, 10, 765-771.

(31) Zhang, H.; Jang, J.; Liu, W.; Talapin, D. V. Colloidal Nanocrystals with Inorganic Halide, Pseudohalide, and Halometallate Ligands. ACS Nano 2014, 8, 7359-7369.

(32) Tang, C. W.; VanSlyke, S. A. Organic Electroluminescent Diodes. Appl. Phys. Lett. 1987, 51, 913.

(33) Burroughes, J. H.; Bradley, D. D. C.; Brown, A. R.; Marks, R. N.; Mackay, K.; Friend, R. H.; Burns, P. L.; Holmes, A. B. Light-Emitting Diodes Based on Conjugated Polymers. Nature 1990, 347, 539-541.

(34) Barford, W. Electronic and Optical Properties of Conjugated Polymers, 1st ed.; Oxford University: New York, 2005.

(35) McGehee, M. D.; Bergstedt, T.; Zhang, C.; Saab, A. P.; O’Regan, M. B.; Bazan, G. C.; Srdanov, V. I.; Heeger, A. J. Narrow Bandwidth Luminescence from Blends with Energy Transfer from Semiconducting Conjugated Polymers to Europium Complexes. Adv. Mater. 1999, 11, 1349-1354.

(36) Coe, S.; Woo, W.-K.; Bawendi, M.; Bulović, V. Electroluminescence from Single Monolayers of Nanocrystals in Molecular Organic Devices. Nature 2002, 420, 800-803.

(37) Huynh, W. U.; Dittmer, J. J.; Alivisatos, A. P. Hybrid NanorodPolymer Solar Cells. Science 2002, 295, 2425-2427.

(38) Shirasaki, Y.; Supran, G. J.; Bawendi, M. G.; Bulović, V. Emergence of Colloidal Quantum-Dot Light-Emitting Technologies. Nat. Photonics 2012, 7, 13-23.

(39) Nizamoglu, S.; Guzelturk, B.; Jeon, D.-W.; Lee, I.-H.; Demir, H. V. Efficient Nonradiative Energy Transfer from InGaN/GaN Nanopillars to CdSe/ZnS Core/Shell Nanocrystals. Appl. Phys. Lett. 2011, 98,163108

(40) Guzelturk, B.; Hernandez Martinez, P. L.; Sharma, V. K.; Coskun, Y.; Ibrahimova, V.; Tuncel, D.; Govorov, A. O.; Sun, X. W.; Xiong, Q.; Demir, H. V.; et al. Study of Exciton Transfer in Dense Quantum Dot Nanocomposites. Nanoscale 2014, 6, 11387-11394.

(41) Chang, T.-W. F.; Musikhin, S.; Bakueva, L.; Levina, L.; Hines, M. A.; Cyr, P. W.; Sargent, E. H. Efficient Excitation Transfer from Polymer to Nanocrystals. Appl. Phys. Lett. 2004, 84, 4295.

(42) Anni, M.; Manna, L.; Cingolani, R.; Valerini, D.; Cretí, A.; Lomascolo, M. Förster Energy Transfer from Blue-Emitting Polymers 
to Colloidal CdSe/ZnS Core Shell Quantum Dots. Appl. Phys. Lett. 2004, 85, 4169.

(43) Warner, J. H.; Watt, A. R.; Thomsen, E.; Heckenberg, N.; Meredith, P.; Rubinsztein-Dunlop, H. Energy Transfer Dynamics of Nanocrystal-Polymer Composites. J. Phys. Chem. B 2005, 109, 90019005.

(44) Hong, S.-K. Energy Transfer by Resonant Dipole-Dipole Interaction from a Conjugated Polymer to a Quantum-Dot. Phys. E 2005, 28, 66-75.

(45) Odoi, M. Y.; Hammer, N. I.; Sill, K.; Emrick, T.; Barnes, M. D. Observation of Enhanced Energy Transfer in Individual Quantum Dot-Oligophenylene Vinylene Nanostructures. J. Am. Chem. Soc. 2006, 128, 3506-3507.

(46) Kaufmann, S.; Stoferle, T.; Moll, N.; Mahrt, R. F.; Scherf, U.; Tsami, A.; Talapin, D. V.; Murray, C. B. Resonant Energy Transfer within a Colloidal Nanocrystal Polymer Host System. Appl. Phys. Lett. 2007, 90, 071108.

(47) Chin, P. T. K.; Hikmet, R. A. M.; Janssen, R. A. J. Energy Transfer in Hybrid Quantum Dot Light-Emitting Diodes. J. Appl. Phys. 2008, 104, 013108.

(48) Lutich, A. A.; Jiang, G.; Susha, A. S.; Rogach, A. L.; Stefani, F. D.; Feldmann, J. Energy Transfer versus Charge Separation in Type-II Hybrid Organic-Inorganic Nanocomposites. Nano Lett. 2009, 9, 2636-2640.

(49) Lutich, A. A.; Poschl, A.; Jiang, G.; Stefani, F. D.; Susha, A. S.; Rogach, A. L.; Feldmann, J. Efficient Energy Transfer in Layered Hybrid Organic/Inorganic Nanocomposites: A Dual Function of Semiconductor Nanocrystals. Appl. Phys. Lett. 2010, 96, 083109.

(50) Jiang, G.; Susha, A. S.; Lutich, A. A.; Stefani, F. D.; Feldmann, J.; Rogach, A. L. Cascaded FRET in Conjugated Polymer/Quantum Dot/ Dye-Labeled DNA Complexes for DNA Hybridization Detection. ACS Nano 2009, 3, 4127-4131.

(51) Stoferle, T.; Scherf, U.; Mahrt, R. F. Energy Transfer in Hybrid Organic/Inorganic Nanocomposites. Nano Lett. 2009, 9, 453-456.

(52) Guzelturk, B.; Martinez, P. L. H.; Zhang, Q.; Xiong, Q.; Sun, H.; Sun, X. W.; Govorov, A. O.; Demir, H. V. Excitonics of Semiconductor Quantum Dots and Wires for Lighting and Displays. Laser Photon. Rev. 2014, 8, 73-93.

(53) Rogach, A. L.; Klar, T. A.; Lupton, J. M.; Meijerink, A.; Feldmann, J. Energy Transfer with Semiconductor Nanocrystals. J. Mater. Chem. 2009, 19, 1208.

(54) Bhattacharyya, S.; Patra, A. Interactions of $\pi$-Conjugated Polymers with Inorganic Nanocrystals. J. Photochem. Photobiol., C 2014, 20, 51-70.

(55) Reiss, P.; Couderc, E.; De Girolamo, J.; Pron, A. Conjugated Polymers/Semiconductor Nanocrystals Hybrid Materials-Preparation, Electrical Transport Properties and Applications. Nanoscale 2011, $3,446-489$.

(56) Clapp, A. R.; Medintz, I. L.; Fisher, B. R.; Anderson, G. P.; Mattoussi, H. Can Luminescent Quantum Dots Be Efficient Energy Acceptors with Organic Dye Donors? J. Am. Chem. Soc. 2005, 127, 1242-1250.

(57) Tessler, N.; Medvedev, V.; Kazes, M.; Kan, S.; Banin, U. Efficient Near-Infrared Polymer Nanocrystal Light-Emitting Diodes. Science 2002, 295, 1506-1508.

(58) Bakueva, L.; Konstantatos, G.; Levina, L.; Musikhin, S.; Sargent, E. H. Luminescence from Processible Quantum Dot-Polymer Light Emitters 1100-1600 nm: Tailoring Spectral Width and Shape. Appl. Phys. Lett. 2004, 84, 3459.

(59) Javier, A.; Meulenberg, R. W.; Yun, C. S.; Strouse, G. F. Photothermal Melting and Energy Migration in Conjugated Oligomer Films Doped with CdSe Quantum Dots. J. Phys. Chem. B 2005, 109, 6999-7006.

(60) Guo, Z.-S.; Zhao, L.; Pei, J.; Zhou, Z.-L.; Gibson, G.; Brug, J.; Lam, S.; Mao, S. S. CdSe/ZnS Nanoparticle Composites with AmineFunctionalized Polyfluorene Derivatives for Polymeric Light-Emitting Diodes: Synthesis, Photophysical Properties, and the Electroluminescent Performance. Macromolecules 2010, 43, 1860-1866.
(61) Kaufmann, S.; Stöferle, T.; Moll, N.; Mahrt, R. F.; Scherf, U.; Tsami, A.; Talapin, D. V.; Murray, C. B. Resonant Energy Transfer within a Colloidal Nanocrystal Polymer Host System. Appl. Phys. Lett. 2007, 90, 071108.

(62) Kwak, J.; Bae, W. K.; Zorn, M.; Woo, H.; Yoon, H.; Lim, J.; Kang, S. W.; Weber, S.; Butt, H.-J.; Zentel, R.; et al. Characterization of Quantum Dot/Conducting Polymer Hybrid Films and Their Application to Light-Emitting Diodes. Adv. Mater. 2009, 21, 50225026.

(63) Zorn, M.; Bae, W. K.; Kwak, J.; Lee, H.; Lee, C.; Zentel, R.; Char, K. Quantum Dot-Block Copolymer Hybrids with Improved Properties and Their Application to Quantum Dot Light-Emitting Devices. ACS Nano 2009, 3, 1063-1068.

(64) Guzelturk, B.; Olutas, M.; Delikanli, S.; Kelestemur, Y.; Erden, O.; Demir, H. V. Nonradiative Energy Transfer in Colloidal CdSe Nanoplatelet Films. Nanoscale 2015, 7, 2545-2551.

(65) Meskers, S. C. J.; Hübner, J.; Oestreich, M.; Bässler, H. Dispersive Relaxation Dynamics of Photoexcitations in a Polyfluorene Film Involving Energy Transfer: Experiment and Monte Carlo Simulations. J. Phys. Chem. B 2001, 105, 9139-9149.

(66) Lyons, B.; Monkman, A. The Role of Exciton Diffusion in Energy Transfer between Polyfluorene and Tetraphenyl Porphyrin. Phys. Rev. B 2005, 71, 235201.

(67) Tomczak, N.; Jańczewski, D.; Han, M.; Vancso, G. J. Designer Polymer-Quantum Dot Architectures. Prog. Polym. Sci. 2009, 34, $393-430$.

(68) Holder, E.; Tessler, N.; Rogach, A. L. Hybrid Nanocomposite Materials with Organic and Inorganic Components for OptoElectronic Devices. J. Mater. Chem. 2008, 18, 1064.

(69) Gösele, U.; Hauser, M.; Klein, U.; Frey, R. Diffusion and LongRange Energy Transfer. Chem. Phys. Lett. 1975, 34, 519-522.

(70) Herz, L.; Silva, C.; Grimsdale, A.; Müllen, K.; Phillips, R. TimeDependent Energy Transfer Rates in a Conjugated Polymer GuestHost System. Phys. Rev. B 2004, 70, 165207.

(71) Gulbinas, V.; Mineviciute, I.; Hertel, D.; Wellander, R.; Yartsev, A.; Sundstrom, V. Exciton Diffusion and Relaxation in MethylSubstituted Polyparaphenylene Polymer Films. J. Chem. Phys. 2007, $127,144907$.

(72) Dias, F. B.; Kamtekar, K. T.; Cazati, T.; Williams, G.; Bryce, M. R; Monkman, A. P. Exciton Diffusion in Polyfluorene Copolymer Thin Films: Kinetics, Energy Disorder and Thermally Assisted Hopping. ChemPhysChem 2009, 10, 2096-2104.

(73) Brédas, J.-L.; Silbey, R. Chemistry. Excitons Surf along Conjugated Polymer Chains. Science 2009, 323, 348-349.

(74) Tagliazucchi, M.; Tice, D. B.; Sweeney, C. M.; Morris-Cohen, A. J.; Weiss, E. A. Ligand-Controlled Rates of Photoinduced Electron Transfer in Hybrid CdSe Nanocrystal/Poly(viologen) Films. ACS Nano 2011, 5, 9907-9917.

(75) Bakueva, L.; Musikhin, S.; Hines, M. A.; Chang, T.-W. F.; Tzolov, M.; Scholes, G. D.; Sargent, E. H. Size-Tunable Infrared (1000-1600 nm) Electroluminescence from PbS Quantum-Dot Nanocrystals in a Semiconducting Polymer. Appl. Phys. Lett. 2003, 82, 2895.

(76) Dabbousi, B. O.; Bawendi, M. G.; Onitsuka, O.; Rubner, M. F. Electroluminescence from CdSe Quantum-Dot/Polymer Composites. Appl. Phys. Lett. 1995, 66, 1316.

(77) Li, Y.; Rizzo, A.; Mazzeo, M.; Carbone, L.; Manna, L.; Cingolani, R.; Gigli, G. White Organic Light-Emitting Devices with CdSe/ZnS Quantum Dots as a Red Emitter. J. Appl. Phys. 2005, 97, 113501.

(78) Park, J. H.; Kim, J. Y.; Chin, B. D.; Kim, Y. C.; Kim, J. K.; Park, O. O. White Emission from Polymer/Quantum Dot Ternary Nanocomposites by Incomplete Energy Transfer. Nanotechnology 2004, 15, 1217-1220.

(79) Baldo, M. A.; O’Brien, D. F.; You, Y.; Shoustikov, A.; Sibley, S.; Thompson, M. E.; Forrest, S. R. Highly Efficient Phosphorescent Emission from Organic Electroluminescent Devices. Nature 1998, 395, 151-154. 
(80) Baldo, M.; Thompson, M.; Forrest, S. High-Efficiency Fluorescent Organic Light-Emitting Devices Using a Phosphorescent Sensitizer. Nature 2000, 403, 750-753.

(81) Anikeeva, P. O.; Madigan, C. F.; Coe-Sullivan, S. A.; Steckel, J. S.; Bawendi, M. G.; Bulović, V. Photoluminescence of CdSe/ZnS Core/Shell Quantum Dots Enhanced by Energy Transfer from a Phosphorescent Donor. Chem. Phys. Lett. 2006, 424, 120-125.

(82) Guzelturk, B.; Hernandez Martinez, P. L.; Zhao, D.; Sun, X. W.; Demir, H. V. Singlet and Triplet Exciton Harvesting in the Thin Films of Colloidal Quantum Dots Interfacing Phosphorescent Small Organic Molecules. J. Phys. Chem. C 2014, 118, 25964-25969.

(83) Mutlugun, E.; Guzelturk, B.; Abiyasa, A. P.; Gao, Y.; Sun, X. W.; Demir, H. V. Colloidal Quantum Dot Light-Emitting Diodes Employing Phosphorescent Small Organic Molecules as Efficient Exciton Harvesters. J. Phys. Chem. Lett. 2014, 5, 2802-2807.

(84) Thompson, N. J.; Wilson, M. W. B.; Congreve, D. N.; Brown, P. R.; Scherer, J. M.; Bischof, T. S.; Wu, M.; Geva, N.; Welborn, M.; Voorhis, T. Van; et al. Energy Harvesting of Non-Emissive Triplet Excitons in Tetracene by Emissive PbS Nanocrystals. Nat. Mater. 2014, 13, 1039-1043.

(85) Tabachnyk, M.; Ehrler, B.; Gélinas, S.; Böhm, M. L.; Walker, B. J.; Musselman, K. P.; Greenham, N. C.; Friend, R. H.; Rao, A. Resonant Energy Transfer of Triplet Excitons from Pentacene to PbSe Nanocrystals. Nat. Mater. 2014, 13, 1033-1038.

(86) Wright, M.; Uddin, A. Organic-Inorganic Hybrid Solar Cells: A Comparative Review. Sol. Energy Mater. Sol. Cells 2012, 107, 87-111.

(87) He, M.; Qiu, F.; Lin, Z. Toward High-Performance OrganicInorganic Hybrid Solar Cells: Bringing Conjugated Polymers and Inorganic Nanocrystals in Close Contact. J. Phys. Chem. Lett. 2013, 4, $1788-1796$.

(88) Ren, S.; Chang, L.-Y.; Lim, S.-K.; Zhao, J.; Smith, M.; Zhao, N.; Bulović, V.; Bawendi, M.; Gradecak, S. Inorganic-Organic Hybrid Solar Cell: Bridging Quantum Dots to Conjugated Polymer Nanowires. Nano Lett. 2011, 11, 3998-4002.

(89) Celik, D.; Krueger, M.; Veit, C.; Schleiermacher, H. F.; Zimmermann, B.; Allard, S.; Dumsch, I.; Scherf, U.; Rauscher, F.; Niyamakom, P. Performance Enhancement of CdSe NanorodPolymer Based Hybrid Solar Cells Utilizing a Novel Combination of Post-Synthetic Nanoparticle Surface Treatments. Sol. Energy Mater. Sol. Cells 2012, 98, 433-440.

(90) Liu, Z.; Sun, Y.; Yuan, J.; Wei, H.; Huang, X.; Han, L.; Wang, W.; Wang, H.; Ma, W. High-Efficiency Hybrid Solar Cells Based on Polymer/ $\mathrm{PbS}_{\mathrm{x}} \mathrm{Se}_{1-\mathrm{x}}$ Nanocrystals Benefiting from Vertical Phase Segregation. Adv. Mater. 2013, 25, 5772-5778.

(91) Xu, Z.; Hine, C. R.; Maye, M. M.; Meng, Q.; Cotlet, M. Shell Thickness Dependent Photoinduced Hole Transfer in Hybrid Conjugated Polymer/Quantum Dot Nanocomposites: From Ensemble to Single Hybrid Level. ACS Nano 2012, 6, 4984-4992.

(92) Itskos, G.; Papagiorgis, P.; Tsokkou, D.; Othonos, A.; Hermerschmidt, F.; Economopoulos, S. P.; Yarema, M.; Heiss, W.; Choulis, S. Size-Dependent Charge Transfer in Blends of $\mathrm{PbS}$ Quantum Dots with a Low-Gap Silicon-Bridged Copolymer. Adv. Energy Mater. 2013, 3, 1490-1499.

(93) Tuncel, D.; Demir, H. V. Conjugated Polymer Nanoparticles. Nanoscale 2010, 2, 484.

(94) Kundu, S.; Bhattacharyya, S.; Patra, A. Photoinduced Energy Transfer in Dye Encapsulated Polymer Nanoparticle-CdTe Quantum Dot Light Harvesting Assemblies. Mater. Horiz. 2015, 2, 60-67.

(95) Bjorgaard, J. A.; Köse, M. E. Amplified Quenching of Conjugated Polymer Nanoparticle Photoluminescence for Robust Measurement of Exciton Diffusion Length. J. Appl. Phys. 2013, 113, 203707.

(96) Groff, L. C.; Wang, X.; McNeill, J. D. Measurement of Exciton Transport in Conjugated Polymer Nanoparticles. J. Phys. Chem. C 2013, 117, 25748-25755.

(97) Tian, Z.; Yu, J.; Wu, C.; Szymanski, C.; McNeill, J. Amplified Energy Transfer in Conjugated Polymer Nanoparticle Tags and Sensors. Nanoscale 2010, 2, 1999-2011. 